\title{
A Flow in a Thin Plastic Layer: Generalizations of the Prandtl's Problem
}

\author{
Vagid Kadymov $^{1, *}$ and Elena Yanovskaya ${ }^{2}$ \\ ${ }^{1}$ Moscow State University of Humanities and Economics, RU-107150 Moscow, Russia \\ ${ }^{2}$ Moscow State Technological University "STANKIN", RU-127055, Moscow, Russia
}

\begin{abstract}
The analytical solutions of various generalizations of the classical L.Prandtl's problem are of great interest in studying the problem of straightening plates using uniaxial stretching beyond the elastic limit. The straightening by stretching allows obtaining a high degree of flatness of thin wide strips and sheets of high-strength steels and special alloys, while the straightening by other methods does not provide satisfactory results. Based on Ilyushin's theory of flow in a thin plastic layer, generalizations of the classical Prandtl's problem on plastic strip compression were studied and their solutions were obtained. The planar problem of compression of a plastic strip between two parallel rough planes with accounting for the asymmetry of the conditions on the spreading ends has been solved and the upper estimate of the total compression force of the face-end areas of the plastically stretched strip has been obtained.
\end{abstract}

\section{Introduction}

The classical problem of compression of a plastic strip between rough planes of solids [1-3] keeps drawing attention of the y research community. This problem is closely related to a number physical processes and phenomena including the phenomenon of slippage along the contact surface, the dominance of the spherical segment of the stress tensor over the deviator components in the plastic strip, the commensurability of normal elastic displacements of contacting bodies with the thickness of the strip [3], the effect of " cold welding" and others. On the other hand, this problem is a key to a better understanding of the mechanism of contact interaction between solid and plastically deformable bodies. L. Prandtl first constructed a limiting stress field, which Nadai supplemented with the corresponding flow velocities.

In refs. $[1,2]$ the planar problem of compression of a preheated strip by means of cooler external bodies has been investigated. As a result of intensive heat exchange, contact layers of solidification are formed. In ref. [4], the solution of a planar problem of a plastic layer spreading with inhomogeneous properties on thickness in an isothermal approximation has been derived. In ref. [5] the planar problem of plastic compression of a threelayer strip with piecewise homogeneous and symmetric thickness properties has been solved. In ref. [6] the solution of Prandtl's problem for a weakly inhomogeneous plastic layer with a yield strength has been presented.

Based on the analysis of the Prandtl - Nadai solution, A.A. Ilyushin proposed a hypotheses, using which he has developed a theory of flow in a thin plastic layer [1]. This theory is based an approximate, averaged over the thickness of the current layer, two-dimensional mathematical model [1] of the spreading process of plastic layers, described by nonlinear partial differential equations of the second order. In more recent studies [7, 8], this theory was generalized to include the case of the flow of a plastic layer on surfaces with anisotropy of properties with respect to the forces of contact friction. In the present work, we develop a newer formulation of the aforementioned models and obtain new results regarding phenomena being studied.

\section{The plane problem of compression of a plastic strip and its solution}

Below on the basis of the averaged (in terms of the thickness of the current layer) theory of flow in a thin plastic layer [1], we present the solutions to various generalizations of the Prandtl's problem of free spreading of a strip enclosed between two parallel planes converging along the normal and relating to external bodies., and occupying a region

$$
S(x, y)=\left\{(x, y) \mid l_{1}(t)<x<l_{2}(t) ; 0 \leq y \leq h(t)\right\} .
$$

Differential equations of the problem have the form [1]:

$$
\frac{\partial u}{\partial x}-\frac{d \lambda}{d t}=0, \quad \frac{\partial p}{\partial x}=-\frac{2 \tau_{s}}{h} \frac{u}{|u|}
$$

where $h=h(t)$ is a well-known law of change of the thickness of plastic layer, occupying the area $S(x, y)=\left\{(x, y) \mid l_{1}(t)<x<l_{2}(t) ; 0 \leq y \leq h(t)\right\}$; 
$\lambda(t)=\ln \left(h_{0} / h(t)\right) \geq 0$ is the degree of deformation by A. A. Ilyushin; $u=u(x, t) ; p=p(x, t)$ is the rate of flow along the layer and contact pressure in the strip. Boundary conditions at free ends:

$$
x=l_{1}: p=\sigma_{s} ; \quad x=l_{2}: p=\sigma_{s},
$$

As is known [1], there is an unknown ramification line of the flow $x=x_{0} \in\left(l_{1}, l_{2}\right)$, in which $u\left(x_{0}, t\right)=0$.

Integrating the equation of quasistatic equilibrium (1) with consideration of the boundary conditions (2), we determine the contact pressure in the layer (Fig.1):

$$
p(x, t)=\left\{\begin{array}{ll}
\sigma_{s}+\frac{2 \tau_{s}}{h}\left(l_{2}-x\right), & x \in\left(x_{0}, l_{2}\right) \\
\sigma_{s}+\frac{2 \tau_{s}}{h}\left(x-l_{1}\right), & x \in\left(l_{1}, x_{0}\right)
\end{array},\right.
$$

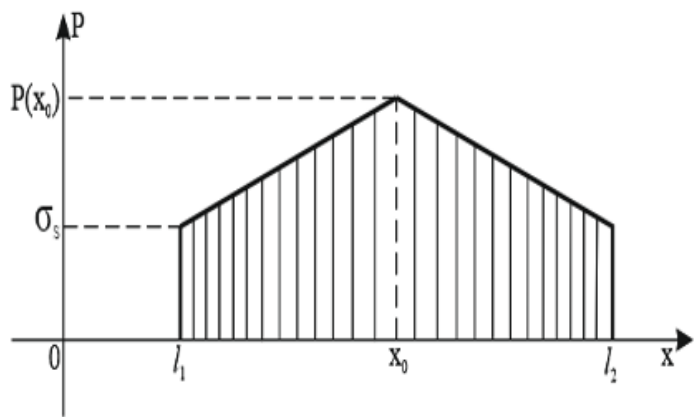

Fig.1. Contact pressure distribution in the problem of plastic layer free spreading.

Integrating the incompressibility equation (1) with consideration of the condition on the ramification line, we find:

$$
u(x, t)=-\frac{1}{h} \frac{d h}{d t}\left(x-x_{0}\right)=-\frac{1}{h} \frac{d h}{d t}\left(x-\frac{l_{1}+l_{2}}{2}\right),
$$

in this case, the flow ramification line $x=x_{0}(t) \mathrm{o}$ is determined from the condition of continuity of the contact pressure.

The total required force for the plastic compression of the strip (the area marked in Fig.1):

$$
\begin{gathered}
P(t)=\int_{l_{1}}^{l_{2}} p(x, t) d x= \\
=\int_{l_{1}}^{x_{0}}\left[\sigma_{s}+\frac{2 \tau_{s}}{h}\left(x-l_{1}\right)\right] d x+\int_{x_{0}}^{l_{2}}\left[\sigma_{s}+\frac{2 \tau_{s}}{h}\left(l_{2}-x\right)\right] d x= \\
=\sigma_{s}\left(l_{2}-l_{1}\right)+\frac{2 \tau_{s}}{h} \frac{\left(l_{2}-l_{1}\right)^{2}}{4},
\end{gathered}
$$

\section{Results. Discussion}

Below we consider the various generalizations of the problem of the free spreading of a plastic layer.

1) The strip spreads freely in both directions so that the contact area of the tool with the plastic strip expanding in both directions, forms a segment $\left[l_{1}(t), l_{2}(t)\right]$ with movable ends (Fig.2).

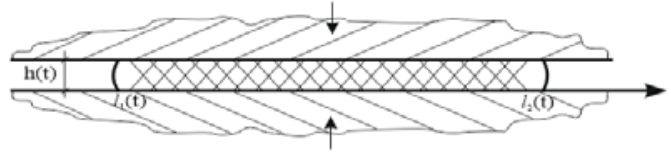

Fig.2. Free spreading of the strip with movable ends.

In this case the free flowing ends of the strip are found from the solution of the following Cauchy problem for a system of linear homogeneous differential equations:

$$
\begin{gathered}
\frac{d l_{2}}{d t}=u\left(l_{2}, t\right)=-\frac{1}{h} \frac{d h}{d t}\left(\frac{l_{2}-l_{1}}{2}\right), \\
\frac{d l_{1}}{d t}=u\left(l_{1}, t\right)=-\frac{1}{h} \frac{d h}{d t}\left(\frac{l_{1}-l_{2}}{2}\right)=\frac{1}{h} \frac{d h}{d t}\left(\frac{l_{2}-l_{1}}{2}\right), \\
t=t_{0}: \quad l_{1}(t)=l_{10}, \quad l_{2}(t)=l_{20}
\end{gathered}
$$

Below we write the solution of the problem (6):

$$
\begin{aligned}
& l_{2}(t)=\frac{1}{2}\left[\left(l_{20}+l_{10}\right)+\frac{h_{0}}{h(t)}\left(l_{20}-l_{10}\right)\right], \\
& l_{1}(t)=\frac{1}{2}\left[\left(l_{20}+l_{10}\right)-\frac{h_{0}}{h(t)}\left(l_{20}-l_{10}\right)\right],(7)
\end{aligned}
$$

2) The plastic strip settles and occupies the contact area with the fixed left end so that it can flow freely in both directions, and represents a segment $\left[l_{10}, l_{2}(t)\right]$ with one movable end (Fig.3). At the same time we make an assumption, that plastic material can flow out from the left end of the contact area.

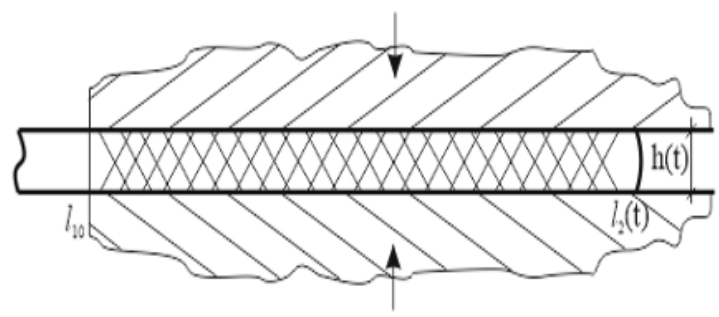

Fig.3. Plastic spreading of the strip with movable right end. 
In this case the above solution remains valid, and only the differential problem for determination of the $l_{2}(t)$ changes:

$$
\begin{gathered}
l_{1}(t)=l_{10}, \quad \frac{d l_{2}}{d t}=u\left(l_{2}, t\right)=-\frac{1}{h} \frac{d h}{d t}\left(\frac{l_{2}-l_{10}}{2}\right), \\
t=t_{0}: \quad l_{2}(t)=l_{20} .
\end{gathered}
$$

The solution of the problem (8) has the form:

$$
l_{2}(t)=l_{10}+\left(l_{20}-l_{10}\right) \sqrt{\frac{h_{0}}{h(t)}},
$$

3) Let the left fixed end of the contact area be pinched so that the plastic flow in the area $\left[l_{10}, l_{2}(t)\right]$ occurs in one direction, and $x_{0} \equiv l_{10}$. In this case, the solution to the boundary value problem

$$
\begin{gathered}
\frac{\partial u}{\partial x}-\frac{d \lambda}{d t}=0, \quad \frac{\partial p}{\partial x}=-\frac{2 \tau_{s}}{h}, \\
x=l_{10}: u\left(l_{10}, t\right)=0 ; \quad x=l_{2}(t): p=\sigma
\end{gathered}
$$

has the form (Fig.4):

$$
\begin{gathered}
p(x, t)=\sigma_{s}+\frac{2 \tau_{s}}{h}\left(l_{2}-x\right), \quad u(x, t)=-\frac{1}{h} \frac{d h}{d t}\left(x-l_{10}\right), \\
l_{2}(t)=l_{10}+\frac{h_{0}}{h}\left(l_{20}-l_{10}\right)
\end{gathered}
$$

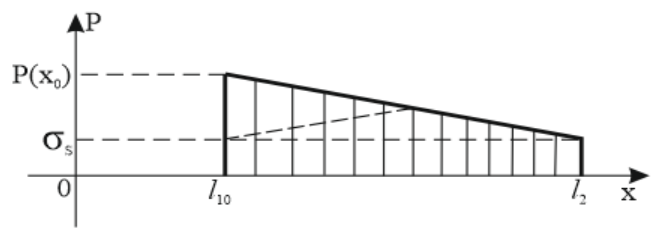

Fig.4. Distribution of contact pressure in the problem of the spreading of a plastic strip with a pinched left end.

The value of the total force required for compressing of the plastic strip in this case certainly depends on the conditions at the left end, and takes the form:

$$
\begin{gathered}
P(t) \equiv P_{3}(t)=\int_{l_{10}}^{l_{2}}\left[\sigma_{s}+\frac{2 \tau_{s}}{h}\left(l_{2}-x\right)\right] d x=, \\
=\sigma_{s}\left(l_{2}-l_{10}\right)+\frac{2 \tau_{s}}{h} \frac{\left(l_{2}-l_{10}\right)^{2}}{2},(10)
\end{gathered}
$$

4) A tensile force is applied at the left (fixed) end of the contact area:

$$
x=l_{10}: \sigma_{x x}=-q_{0}>0,
$$

so that $-\sigma_{s}<q_{0}<0$. According to the condition of full plasticity ( $p=q+\sigma_{s}$ ) [1], we have the condition for the contact pressure $p=q_{0}+\sigma_{s}$ at the left end:

$$
0<p<\sigma_{s} \text {. }
$$

Let us single out the limiting case:

$$
q_{0}=-\sigma_{s} \text {, }
$$

which simulates the process of the plastic stretching of a thin strip with simultaneous plastic pressing of its" clamped " end parts.

The solution of the above simple boundary value problem in this case takes the form (Fig.5):

$$
\begin{gathered}
p(x, t)=\left\{\begin{array}{cc}
\sigma_{s}+\frac{2 \tau_{s}}{h}\left(l_{2}-x\right), \quad x \in\left(x_{0}, l_{2}\right) \\
\sigma_{s}+q_{0}+\frac{2 \tau_{s}}{h}\left(x-l_{10}\right), & x \in\left(l_{10}, x_{0}\right)
\end{array},\right. \\
u(x, t)=-\frac{1}{h} \frac{d h}{d t}\left(x-x_{0}\right), \quad(11)
\end{gathered}
$$

where

$$
x_{0}=\frac{l_{10}+l_{2}}{2}-\frac{q_{0}}{4 \tau_{s}} h \geq \frac{l_{10}+l_{2}}{2} .
$$

The value of the total force required for the plastic compressing in this case takes the form (the shaded area on Fig.5):

$$
\begin{gathered}
P(t)=\int_{l_{10}}^{l_{2}} p(x, t) d x==\left.\left[\left(\sigma_{s}+q_{0}\right) x+\frac{2 \tau_{s}}{h}\left(\frac{x^{2}}{2}-l_{10} x\right)\right]\right|_{l_{10}} ^{x_{0}}+ \\
+\left.\left[\sigma_{s} x+\frac{2 \tau_{s}}{h}\left(l_{2} x-\frac{x^{2}}{2}\right)\right]\right|_{x_{0}} ^{l_{2}}=\sigma_{s}\left(l_{2}-l_{10}\right)+\frac{2 \tau_{s}}{h} \frac{\left(l_{2}-l_{10}\right)^{2}}{4}+ \\
+q_{0}\left(\frac{l_{2}+l_{10}}{2}-\frac{q_{0}}{2 \tau_{s}} h\right) \leq P_{1}(t)
\end{gathered}
$$

where $P_{1}(t)=\sigma_{s}\left(l_{2}-l_{10}\right)+\frac{2 \tau_{s}}{h} \frac{\left(l_{2}-l_{10}\right)^{2}}{4}$ is the total force required for the plastic pressing of the strip with free ends. 


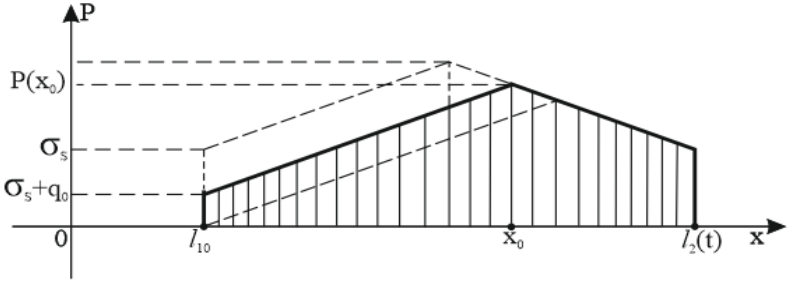

Fig.5. The distribution of contact pressure in the plastic strip (at the left end of which a tensile force $\sigma_{x x}=-q_{0}>0$ is applied).

\section{Conclusion}

The analytical solutions of various generalizations of the classical L.Prandtl problem are of considerable interest in studying of the problem of straightening plates using uniaxial stretching beyond the elastic limit.

As shown here, straightening by stretching allows to obtain a high degree of flatness of thin wide strips and sheets of high-strength steels and special alloys, while the straightening with other methods does not provide such good y results.

The above-mentioned solutions of L.Prandtl planar problem can be extended to the space contact problems of flow in a thin plastic layer between the converging surfaces of solid (elastically deformable) external bodies.
This work was carried out using equipment provided by the Center of Collective Use of MSUT "STANKIN".

\section{References}

1. A.A. Ilyushin, Proceedings 2, (Plasticity, M.:Fizmatlit. 2004).

2. L. Prandtl, ZAMM, 3(6), 401-406 (1923)

3. I.A. Kiyko, Theory of plastic flow (M.: MSU. 1978).

4. A.I. Kuznetsov, Mech. Stos. 2. 12, 163-171 (1960)

5. V. Kadymov, R. Wille, ZAMM, 75(S1), 293294 (1995)

6. D.V. Georgiyevsky, Mechanics of solids, 1, 4759 (2006)

7. V. Kadymov, Nonlinear Analysis: Theory \& Appl., 30(8), 5259-5265 (1997)

8. I.A. Kiyko, Applied mathematics and mechanics, 70, 344-351 (2006)

9. V. Kadymov, Mathematical Methods in the Applied Sciences, 38(16), 3421-3431 (2015)

10. V.A. Kadymov, Mathematical modeling of contact problems of plastic flow, (Monograph. Germany: Palmarium Academic Publishing, 2016) 